Baar, Robert; Hartmann, Jutta; Kampshoff, Marita

\title{
Geschlechterreflektierte Professionalisierung - Geschlecht und Professionalität in pädagogischen Berufen. Eine Einführung
}

Baar, Robert [Hrsg.]; Hartmann, Jutta [Hrsg.]; Kampshoff, Marita [Hrsg.]: Geschlechterreflektierte Professionalisierung. Geschlecht und Professionalität in pädagogischen Berufen. Opladen ; Berlin ; Toronto : Verlag Barbara Budrich 2019, S. 31-54. - (Jahrbuch erziehungswissenschaftliche Geschlechterforschung; 15)

Quellenangabe/ Reference:

Baar, Robert; Hartmann, Jutta; Kampshoff, Marita: Geschlechterreflektierte Professionalisierung Geschlecht und Professionalität in pädagogischen Berufen. Eine Einführung - In: Baar, Robert [Hrsg.]; Hartmann, Jutta [Hrsg.]; Kampshoff, Marita [Hrsg.]: Geschlechterreflektierte Professionalisierung. Geschlecht und Professionalität in pädagogischen Berufen. Opladen ; Berlin ; Toronto : Verlag Barbara Budrich 2019, S. 31-54 - URN: urn:nbn:de:0111-pedocs-219026 - DOI: 10.25656/01:21902

https://nbn-resolving.org/urn:nbn:de:0111-pedocs-219026

https://doi.org/10.25656/01:21902

in Kooperation mit / in cooperation with:

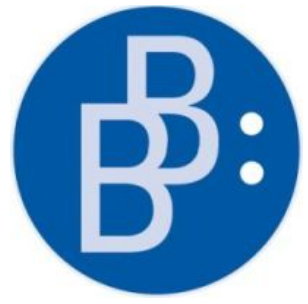

https://www.budrich.de

\section{Nutzungsbedingungen}

Gewährt wird ein nicht exklusives, nicht übertragbares, persönliches und beschränktes Recht auf Nutzung dieses Dokuments. Dieses Dokument ist ausschließlich für den persönlichen, nicht-kommerziellen Gebrauch bestimmt. Die Nutzung stellt keine Übertragung des Eigentumsrechts an diesem Dokument dar und gilt vorbehaltlich der folgenden Einschränkungen: Auf sämtlichen Kopien dieses Dokuments müssen alle Urheberrechtshinweise und sonstigen Hinweise auf gesetzlichen Schutz beibehalten werden. Sie dürfen dieses Dokument nicht in irgendeiner Weise abändern, noch dürfen Sie dieses Dokument für öffentliche oder kommerzielle Zwecke vervielfältigen, öffentlich ausstellen, aufführen, vertreiben oder anderweitig nutzen.

Mit der Verwendung dieses Dokuments erkennen Sie die Nutzungsbedingungen an.

\section{Terms of use}

We grant a non-exclusive, non-transferable, individual and limited right to using this document.

This document is solely intended for your personal, non-commercial use. Use of this document does not include any transfer of property rights and it is conditional to the following limitations: All of the copies of this documents must retain all copyright information and other information regarding legal protection. You are not allowed to alter this document in any way, to copy it for public or commercial purposes, to exhibit the document in public, to perform, distribute or otherwise use the document in public.

By using this particular document, you accept the above-stated conditions of use.

\section{Kontakt / Contact:}

\section{peDOCS}

DIPF | Leibniz-Institut für Bildungsforschung und Bildungsinformation Informationszentrum (IZ) Bildung

E-Mail: pedocs@dipf.de

Internet: www.pedocs.de

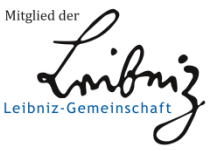


Robert Baar

Jutta Hartmann

Marita Kampshoff (Hrsg.)

\section{$15 / 2019$}

Jahrbuch erziehungswissenschaftliche Geschlechterforschung

Geschlechterreflektierte Professionalisierung Geschlecht und Professionalität in pädagogischen Berufen 


\section{Robert Baar}

Jutta Hartmann

Marita Kampshoff (Hrsg.)

\section{Geschlechterreflektierte Professionalisierung - Geschlecht und Professionalität in pädagogischen Berufen}

Verlag Barbara Budrich Opladen • Berlin • Toronto 2019 
Bibliografische Information der Deutschen Nationalbibliothek

Die Deutsche Nationalbibliothek verzeichnet diese Publikation in der Deutschen

Nationalbibliografie; detaillierte bibliografische Daten sind im Internet über

http://dnb.d-nb.de abrufbar.

Gedruckt auf säurefreiem und alterungsbeständigem Papier.

Alle Rechte vorbehalten.

(C) 2019 Verlag Barbara Budrich, Opladen, Berlin \& Toronto

www.budrich-verlag.de

ISBN 978-3-8474-2277-8 (Paperback)

eISBN 978-3-8474-1310-3 (eBook)

Das Werk einschließlich aller seiner Teile ist urheberrechtlich geschützt. Jede Verwertung außerhalb der engen Grenzen des Urheberrechtsgesetzes ist ohne Zustimmung des Verlages unzulässig und strafbar. Das gilt insbesondere für Vervielfältigungen, Übersetzungen, Mikroverfilmungen und die Einspeicherung und Verarbeitung in elektronischen Systemen.

Typographisches Lektorat: Anja Borkam, Jena

Umschlaggestaltung: disegno visuelle kommunikation, Wuppertal - www.disenjo.de Druck: paper \& tinta, Warschau

Printed in Europe 


\section{Inhalt}

\section{Einleitung}

Robert Baar, Jutta Hartmann \& Marita Kampshoff

Vorwort. 7

Robert Baar, Jürgen Budde, Marita Kampshoff \& Astrid Messerschmidt Redaktion des Jahrbuchs erziehungswissenschaftliche

Geschlechterforschung

Von der Frauen- und Geschlechterforschung in der

Erziehungswissenschaft zur erziehungswissenschaftlichen

Geschlechterforschung....

Maja S. Maier

Erziehungswissenschaftliche Geschlechterforschung?

Ein Essay zu Verhältnisbestimmung und Forschungsprogrammatik 15

Robert Baar, Jutta Hartmann \& Marita Kampshoff

Geschlechterreflektierte Professionalisierung - Geschlecht und

Professionalität in pädagogischen Berufen. Eine Einführung

\section{Vielfältige Lebensweisen im Fokus von Professionalität}

Florian Cristobal Klenk

Interdependente Geschlechtervielfalt als un/be/deutende Anforderung an pädagogische Professionalität

Mart Busche \& Uli Streib-Brzič

Die Entwicklung heteronormativitätskritischer Professionalität in

Reflexions-Workshops - Zur Verbindung von pädagogischem

Erfahrungswissen und wissenschaftlichem Erkenntniswissen im Kontext

von Praxisforschung

Carolin Vierneisel \& Johannes Nitschke

(De-)Professionalisierungstendenzen?! Vielfalts*sensible Bildung im

Lehramtsstudium. 


\section{Geschlechterreflektierte Professionalität im Elementarbereich}

\section{Melanie Kubandt}

Ansprüche an ein geschlechtergerechtes, professionelles Handeln im Elementarbereich - Ethnographische Perspektiven auf Ungewissheiten,

Komplexitäten und Grenzen im pädagogischen Alltag

Susann Fegter, Anna Hontschik, Eszter Kadar, Kim-Patrick Sabla \& Maxine Saborowski

Bezüge auf Familie als Moment der Vergeschlechtlichung pädagogischer Professionalität: Diskursanalytische Perspektiven auf Äußerungen in Gruppendiskussionen mit Kita-Teams

\section{Herausforderungen sexualpädagogischer Professionalisierung}

\section{Anja Eichhorn}

Doing Sexual Agency: Sexuelle Handlungsfähigkeit sexuell

missbrauchter jugendlicher Mädchen in der stationären Jugendhilfe

Marion Thuswald

Geschlechterreflektierte sexuelle Bildung?

Heteronormativität und Verletzbarkeit als Herausforderungen

sexualpädagogischer Professionalisierung

\section{Rezension}

Marina Dangelat, Frauke Grenz \& Christine Thon

Rezension zu: Gesicht Zeigen! (2017) (Hrsg.): „Weiße können nicht rappen“. Das Positionierungsspiel gegen Vorurteile und Klischees und zu: Wedl, Juliette (2018): Identitätenlotto. Ein Spiel quer durchs Leben ... 183

Verzeichnis der Autor_innen 191 


\section{Geschlechterreflektierte Professionalisierung - Geschlecht und Professionalität in pädagogischen Berufen. Eine Einführung.}

Die erziehungswissenschaftlichen Diskurse um Professionalität und um Geschlecht weisen einige Parallelen auf: Beide Diskurse sind höchst aktuell und blicken doch auf eine lange Tradition zurück, in beiden Bereichen gibt es unterschiedliche Positionen und Theorien, die kontrovers, zuweilen auch konfrontativ Anspruch auf Geltung erheben, und beiden ist gemein, dass es sich bislang als schwieriges Unterfangen darstellt, eine Brücke zwischen Theorie und Praxis zu schlagen, d.h., wissenschaftliche Erkenntnisse für den pädagogischen Alltag nutzbar zu machen und sie in diesen zu transferieren wie aber auch Impulse aus der Praxis theorieprüfend bzw. -revidierend aufzugreifen.

So lassen sich beispielsweise die Fragen stellen, warum es vielen Lehrkräften kaum gelingt, gut erforschte Strategien des classroom managements zur (professionellen) Prävention von Unterrichtsstörungen (vgl. bereits Kounin 1976) im Unterricht anzuwenden, oder warum im sozialpädagogischen Kontext teilweise immer noch Angebote, wie die pädagogische Begleitung von Girls' und Boys' Days ausgearbeitet werden, die weniger einer reflektierten Mädchen- und Jungenarbeit folgen, sondern vielmehr einem essentialistisch ausgerichteten Ansatz entsprechen (vgl. kritisch bspw. Budde et al. 2011). Sie tun dies, obwohl im erziehungswissenschaftlichen Diskurs längst differenziert Ansätze des Doing Gender, der Dekonstruktion und der Performativität vertreten werden (vgl. bspw. Fritzsche et al. 2001; Faulstich-Wieland 2004; Hartmann 2004; Tervooren 2006) sowie Weiblichkeit und Männlichkeit nicht nur im sozialwissenschaftlichen Diskurs im Plural (vgl. bspw. Connell 2000; Scholz 2010) und unter intersektionalen Vorzeichen (vgl. bspw. Dietze et al. 2007; Winker/Degele 2009; Busche et al. 2010; Busche/Cremers 2012; Tuider 2012; Emmerich/Hormel 2013; Walgenbach 2014; Hartmann 2017a) gedacht werden.

Gleichzeitig ist kritisch zu fragen, warum auch die erziehungswissenschaftliche Frauen- und Geschlechterforschung so lange gebraucht hat, um beispielsweise vielfältige geschlechtliche und sexuelle Lebensweisen, die, ausgehend von der Emanzipationsbewegung von Lesben und Schwulen, seit Mitte der 1980er Jahren in Aufklärungsprojekten in Schulen entlang unterschiedlicher Konzepte thematisiert werden, als relevanten Bildungsinhalt zu erkennen, zu 
berücksichtigen und zu beforschen (vgl. bspw. Hartmann et al. 1998; Hartmann 2002, Timmermanns 2003). Auch in Bezug auf allgemeindidaktische Themenstellungen erscheint es verwunderlich, dass beispielsweise der Einbezug außerschulischer Lernorte in den Unterricht seit langer Zeit zum festen methodischen Repertoire der Schulen gehört und es zwar zahlreiche Konzepte und Begründungstheorien zu diesem Lernsetting gibt, die Forschungslage zu den Praktiken vor Ort oder zu deren Effekten vor dem Hintergrund von Heterogenität und Diversität der Lernenden aber als ausgesprochen unbefriedigend bezeichnet werden kann - obwohl gerade letzterer in den vorliegenden Begründungstheorien immer wieder in Anschlag gebracht wird (vgl. Baar/Schönknecht 2018).

\section{Professionalität-zwischen Persönlichkeitsparadigma und Professionali- sierungsansätzen}

Im erziehungswissenschaftlichen Kontext grenzt sich der Professionalisierungsansatz vom Persönlichkeitsparadigma ab: Professionalität als Voraussetzung für ,gutes', d.h. wirksames pädagogisches Handeln, erscheint nicht naturgegeben, sondern ist an Kompetenzen, Haltungen und einen reflexiven wie forschenden Habitus gebunden, die anzubahnen und auszubilden Aufgabe der Vorbereitung auf den Beruf (an Universitäten, Hochschulen oder Fachschulen) ist (vgl. bspw. Lipowsky 2006) und die es im Berufsleben zur Entfaltung zu bringen und lebendig zu halten gilt. Professionalität wird damit als plan-, vermittel- und erlernbar deklariert; sie kann entwickelt werden und hat sich vor dem Hintergrund beruflicher Erfahrung weiterzuentwickeln.

Ganz anders argumentierten Anhänger_innen des Persönlichkeitsparadigmas, wie historisch beispielsweise Spranger (1958: 6), der dem ,geborenen', dem ,echte[n] Erzieher [...] ein ursprüngliches Organ für die Bahnen“" zuschreibt, ,in denen der durch ihn hindurchwirkende Geist weht“. Spranger bewegt sich damit in der Tradition vieler reformpädagogischer Ansätze. So schrieb Kerschensteiner, ein Vertreter der Arbeitsschulbewegung: „Der Erzieher ist eine im geistigen Dienst der Gemeinschaft stehende Lebensform des sozialen Grundtyps, die aus reiner Neigung zum werdenden, unmündigen Menschen als einem eigenartigen zukünftigen Träger zeitloser Werte dessen seelische Gestaltung nach Maßgaben seiner besonderen Bildsamkeit in dauernder Bestimmtheit zu beeinflussen imstande ist und in der Bestätigung dieser Neigung seine höchste Befriedigung findet" (Kerschensteiner 1921: 48f.). Es 
geht in diesen Beispielen um Persönlichkeitsmerkmale, um (angeborene) Charaktereigenschaften, die einen Menschen dazu befähigen, Kinder und Jugendliche zu erziehen und zu bilden. Auch heute noch wird an prominenten Stellen auf das Persönlichkeitsparadigma rekurriert; so zum Beispiel im von der Kommission der Europäischen Gemeinschaft und den Kultusministerien verschiedener Bundesländer geförderten Selbsterkundungsbogen für die Eignung als Lehrperson für angehende Studierende, der personengebundene Eigenschaften wie Kontaktfreudigkeit, Warmherzigkeit, Gewissenhaftigkeit und Prinzipientreue als ,eine solide Basis für den Studien- und den Berufserfolg bei Lehrkräften“(CCT 2018: o. S.) deklariert.

\section{Geschlecht - zwischen essentialistischen und sozial(de)konstruktivistischen Ansätzen}

Betrachtet man den erziehungswissenschaftlichen Diskurs um Geschlecht und Geschlechterverhältnisse, so scheinen sich im Wesentlichen zwei ähnlich gelagerte Grundannahmen gegenüberzustehen: Auf der einen Seite stehen sozial(de)konstruktivistische Perspektiven, die Geschlecht als ein - gesellschaftlich bedingtes und psychisch zum Teil tief verankertes - Tun, als erlernt, erworben, gestalt- und veränderbar beschreiben und somit als eine bedeutende und dringend zu reflektierende Aufgabe im Erziehungs- und Bildungsprozess entwerfen. Zum anderen existiert ein essentialistisches Verständnis von Geschlecht, das auf naturgegebene und natürliche Unterschiede zwischen den Geschlechtern in einem bipolar und heteronormativ gedachten Verhältnis rekurriert. Mit diesem sind - ähnlich wie beim oben skizzierten Persönlichkeitsparadigma - konkrete Vorstellung zu spezifischen Wesenseigenschaften und Erwartungen an ein (geschlechtskonformes) Verhalten verbunden.

Im historischen Erbe lässt sich dies besonders deutlich bei Rousseau aufzeigen: Im fünften Buch Sophie oder die Frau seines Werkes Emile oder Über die Erziehung (1762/1993) bezeichnet er Frauen als passiv und schwach, Männer hingegen als aktiv und stark. Die gesellschaftlichen Aufgaben von Frauen sieht er vor dem Hintergrund ihrer natürlichen Bestimmung auf das Private, auf Haushaltsführung, Kindererziehung und auf die Rolle als Gattin beschränkt, währenddessen er Männern den öffentlichen Raum vorbehält. Die unterschiedlichen und komplementären Wesenseigenschaften sowie Aufgabenbereiche der Geschlechter verlangen Rousseau zufolge nach differenter Erziehung: „Nachdem einmal bewiesen ist, dass der Mann und die Frau weder nach dem Charakter noch nach dem Temperament gleich gebildet sind noch sein dürfen, so folgt daraus, dass sie auch nicht die gleiche Erziehung haben dürfen. Folgen sie den Weisungen der Natur, so handeln sie wohl gemeinsam, 
aber sie dürfen nicht das Gleiche tun" (ebd.: 392). Wissenschaftliche Bildung müsse Männern vorbehalten sein; Frauen, deren ,Fassungskraft [...] Genauigkeit und Aufmerksamkeit" (ebd.: 421) hierfür nicht ausreiche, sollen sich hingegen auf die praktische Anwendung der Erkenntnisse im Privaten beschränken. Rousseaus Werk hatte entscheidenden Einfluss auf den gesellschaftlichen Diskurs, und der von ihm entworfene Katalog weiblicher Tugenden mit seinen obersten Zielen Sanftmut und Folgsamkeit war über lange Zeit prägend für die Mädchenbildung (vgl. Schneider-Taylor 2012). Ganz ähnlich argumentierte der Philanthrop Campe (1798/1988), der Bildung von Mädchen als Mittel verstand, diese auf ihre vermeintliche Bestimmung im Reproduktionsbereich vorzubereiten und das dafür notwendige, von Sanftmut, Bescheidenheit und Unterordnung geprägte Gemüt auszubilden.

Der historisch geführte wissenschaftliche Diskurs um das Wesen und die Bestimmung der Geschlechter kann zwar als grundsätzlich überwunden gelten, wird aber dennoch immer wieder und erneut in Anschlag gebracht, so beispielsweise im Rahmen des Diskurses um die (vermeintliche) Feminisierung des Bildungswesens (vgl. bspw. kritisch Kuhn 2008; Baar 2009), oder aber im Rahmen der grundsätzlichen und in jüngster Zeit zunehmenden Anfeindungen gegenüber den Gender Studies insgesamt (vgl. bspw. kritisch Hark/Villa 2015; Lang/Peters 2018).

Gleichzeitig erweist sich aber auch die soeben aufgespannte Opposition von Essentialisten und (De-)Konstruktivisten als vereinfacht. So tritt der essentialistisch ausgerichtete Diskurs kaum unter diesem Label auf und es wird selten explizit entsprechend historischer Vorlagen argumentiert. Eher finden sich implizite Argumentationsmuster und Diskursmechanismen. Auf diese treffen wir stellenweise selbst in Diskussionsbeiträgen, die sich auf (de)konstruktivistische Theorien beziehen bzw. sich vordergründig in diesen verorten, in den weiteren Konkretisierungen dann jedoch deutlich hinter deren Prämissen zurückfallen (vgl. kritisch Kleiner/Klenk 2017: 98). Zugleich kann ein ,strategischer Essentialismus“ (vgl. Spivak 1996) auch verhelfen, den Zugang zu Ressourcen zu sichern. Bei näherer Betrachtung erweist sich die Opposition zwischen essentialistischen und konstruktivistischen Perspektiven darüber hinaus als den unterschiedlich vorfindbaren, zum Teil sehr komplexen sozialkonstruktivistischen und kulturtheoretischen Theorien nicht gerecht werdend. Zwar gründen deren Zugänge auf der gemeinsamen Annahme, symbolische Ordnungen im Sinne kollektiver Verstehens- und Bedeutungsweisen stifteten die soziale Welt. Doch bestehen bedeutsame Unterschiede in verschiedenen Theorierichtungen - im Mentalismus, im Textualismus, in der Praxeologie hinsichtlich der Konzeption der Art der kulturellen Codes sowie der Art und dem Ort und der Tiefe ihrer Wirksamkeit (vgl. Dekker 2012; Hartmann 2017a). 


\section{Historische Verschränkung der Diskurse und essentialistische Reaktivierungen}

Historisch betrachtet vereinen sich erziehungswissenschaftliches Persönlichkeitsparadigma und essentialistische Sicht auf Geschlecht besonders deutlich im Konzept der ,geistigen Mütterlichkeit', mit dem im wilhelminischen Deutschland Vertreterinnen der ersten westlich-bürgerlichen Frauenbewegung (wie bspw. Helene Lange) Frauen der höheren Stände Zugang zur Arbeitswelt verschaffen wollten (und letztlich auch konnten) (vgl. Baader 2018). Frauen wurden dabei ihnen - und nur ihnen - inhärente Wesenseigenschaften wie Fürsorglichkeit und Hilfsbereitschaft zugeschrieben, die diese als prädestiniert für die Ausübung sozialer Berufe ausweisen würden (vgl. Heite 2009a). Gleichzeitig wurde das Konzept der ,Kulturaufgabe der Frau' entwickelt (vgl. Baader 2018: 17), das Frauen die maternalistisch gedachte Bestimmung für soziale Berufe zuwies. Es sind die mit Weiblichkeit verschweißten Eigenschaften und Fähigkeiten wie Zuwendung und Fürsorge, die nicht nur zu dem hohen Anteil von Frauen in Pädagogik und Sozialer Arbeit führten, sondern zugleich auch die niedrige Bewertung und Entlohnung dieser Tätigkeiten beding(t)en. Dies korrespondiert mit einer ungleichen Verteilung der Geschlechter im gesamten Care-Bereich, dem Feld sorgender, pflegender und erziehender Tätigkeiten, und einem hier nach wie vor anzutreffenden geschlechtshierarchischen Arbeitsmarkt. Mit Blick auf diese Arbeitsfelder ist eine institutionalisierte Ungleichheitsordnung zwischen den Geschlechtern festzustellen, die auch heute noch - wenn auch subtiler - über als natürlich unterstellte Eigenschaften legitimiert wird (vgl. Bereswill 2016: 35).

Ende der 1970er Jahre griffen Ostner \& Beck-Gernsheim (1979) das eine Geschlechterdifferenz essentialistisch begründende Theorem von Anfang des 20. Jahrhunderts wieder auf, indem sie von einem, weiblichen Arbeitsvermögen' sprachen. Sie beschrieben für Frauen und Männer je unterschiedliche Lebenswelten, Anforderungen und Erfahrungen, die - einem sozialisationstheoretischen Verständnis folgend - quasi eine Art Sozialcharakter ausbilden und bei Frauen zu Eigenschaften wie Empathie, Intuition und Reproduktionsbezogenheit führen sollen, die sie im Rahmen der geschlechtlichen Arbeitsteilung für den Haushalts- bzw. haushaltsnahen Bereich prädestinieren. Im aktuelleren Diskurs um das underachievement von Jungen wird die Debatte über ein vergeschlechtlichtes Arbeitsvermögen unter einem anderen Vorzeichen geführt: In der Tradition psychoanalytischer Theorien wird auf die vermeintlich anthropologische Tatsache gleichgeschlechtlicher Identifikation rekurriert, nach der nur Männer gute Pädagogen für Jungen sein können. Lehrer, so beispielsweise Matzner \& Tischner (2012), könnten Jungen schon aufgrund ihrer eigenen Männlichkeit besser verstehen und deren Verhalten nachvollziehen, während der „Harmonisierungsterror“ der Lehrerinnen Jungen daran hindere, „,ich 
[...] optimal zu entwickeln“ (ebd.: 436) (vgl. kritisch Rieske 2011; FaulstichWieland 2012; Fegter 2012; Baar 2014). Einem naturalistischen Verständnis folgend werden Geschlecht und mit ihm verbundene Persönlichkeitsmerkmale zu einem entscheidenden Qualifikationsmerkmal erhoben, das als Garant für Professionalität stehe - oder aber diese verhindere. Die im Rahmen dieses Diskurses zum Ausdruck gebrachte Distinktion und Abwertung des (vermeintlich) Weiblichen zeugen von tiefem Antifeminismus und einem reaktionären Festhalten an patriarchalen Machtstrukturen.

\section{Aktuelle (de-)konstruktivistische Konzeptualisierungen von Geschlecht}

In allgemeinen Konzepten, die suggerieren, geschlechtsneutral zu sein, wirkt Geschlecht nicht nicht. Durch Impulse der Frauenbewegung konnten Ende der 1970er Jahre angestoßene Studien bspw. feststellen, dass Mädchen als Zielgruppe in der offenen Jugendarbeit bis dahin ,quantitativ und qualitativ unbeachtet geblieben" (Bitzan 2004: 462) waren. ,Jugendarbeit ist Jungenarbeit" war zu Beginn der erziehungswissenschaftlichen Frauen- und Geschlechterforschung eine vielzitierte Erkenntnis, die hervorhob, dass die Jugendarbeit sich mit ihren Ressourcen unhinterfragt an einem männlichen Subjekt mit dessen Lebenslagen ausrichtete (vgl. Eggers 2012: 233). Mit dem gemeinsamen Ziel, eine geschlechterreflektierte Professionalisierung voranzutreiben, führ $(\mathrm{t}) \mathrm{en}$ im Anschluss daran unterschiedliche geschlechtertheoretische Positionierungen zur Entwicklung verschiedener, als ,geschlechtersensibel', , geschlechtsbewusst' oder , geschlechtergerecht' benannter Konzepte. Vielfach wurden diese Ansätze ausgehend von einer parteilichen Mädchen- und antisexistischen Jungenarbeit (vgl. bspw. Glücks/Ottemeier-Glücks 1994; Bitzan/Daigler 2004; Forster 2004; Kunert-Zier 2005) sowie von einer sowohl im schulischen wie im außerschulischen Bereich diskutierten reflexiven Koedukation (vgl. bspw. Horstkemper/Faulstich-Wieland 1996) (weiter)entwickelt.

Debatten um doing gender, queere Perspektiven, Performativität und Dekonstruktion führ(t)en dazu, dass aktuell nicht nur das hierarchische Verhältnis zwischen den Geschlechtern, sondern vielmehr auch das zweigeschlechtliche Paradigma, das in der Regel hinter den genannten geschlechterreflektierenden Konzepten steht, in Frage gestellt wird. Studien, die einem sozialkonstruktivistischen Ansatz um ein doing gender oder doing difference folgen, blicken nicht länger auf Geschlechterunterschiede, sondern auf die Praktiken der Geschlechterunterscheidungen (vgl. bspw. Breidenstein/Kelle 1998). Für den Bereich Schule liegt mittlerweile eine ganze Reihe von Studien vor, die nicht mehr das Ziel verfolgen, auf Geschlechterdifferenzen hinzuweisen, sondern 
die rekonstruieren, wie Geschlecht, aber auch Ethnie oder Milieu in alltäglichen Interaktionen hervorgebracht wird (vgl. bspw. Faulstich-Wieland et al. 2004; Kalthoff 2006; Budde et al. 2008; Kampshoff 2013). Für Professionalisierungsprozesse sind diese Studien insofern wichtig, als sie für die eigene Beteiligung und Veränderungsmöglichkeiten bei den kleinen und alltäglichen Interaktionen, die oftmals unbewusst ablaufen, sensibilisieren wie für das Eigeninteresse von Kindern, Jugendlichen und Erwachsenen, sich in einer Gesellschaft, in der Zweigeschlechtlichkeit als zentrale Ordnungskategorie wirkt, als Mädchen oder Junge, Frau oder Mann zu positionieren.

Als für die pädagogische Praxis zentral erweist sich weiterhin die Frage, an welchen Geschlechtervorstellungen sich Deutungs- und Zuschreibungspraxen der Pädagog innen im Alltag orientieren. Mit Blick auf den höheren Anteil von Jungen und jungen Männern in den erzieherischen Hilfen läge bspw. ein Missverständnis vor, würde dieser mit unproblematischeren Lebenslagen von Mädchen und jungen Frauen erklärt. Bitzan (2004: 466) spricht von einer gravierenden „Verkennung mädchenspezifischer Probleme“, zu denen insbesondere sexuelle Gewalt zählt. Bis heute wird sexualisiertes Verhalten von Mädchen nicht vor dem Hintergrund bestehender Traumatisierungen, z.B. nach sexuellen Gewalterfahrungen, wahrgenommen und eingeordnet, sondern führt nach wie vor häufig zu Zurückweisung, Verboten und anderen Bestrafungen von Seiten der Fachkräfte (vgl. Schmauch 2016). Konzeptuell setzen hier, wie an vergleichbaren Phänomenen, die Fragen nach geschlechtsbezogenen Normalitätskonstruktionen und Bewältigungsstrategien sowie nach dem differenten ,Mehr' zum vorgegebenen Konstrukt ,Mädchen/Junge' ein. Vormals unhinterfragte konventionelle Übereinkünfte können so zum Gegenstand pädagogischer wie gesellschaftspolitischer Verhandlung werden.

Geschlechtertheoretisch betrachtet ist mit entsprechenden Studien und daran orientierten Konzepten eine Essentialismus- und Homogenisierungskritik verbunden. Studien, die poststrukturalistischen Ansätzen von Performativität und Dekonstruktion folgen, greifen darüber hinaus auch die gendertheoretische Kritik an der Vereindeutigung von Geschlecht in der erziehungswissenschaftlichen Frauen- und Geschlechterforschung auf. Wie Ansätze des doing gender betont der Ansatz der Performativität die Herstellung von Geschlecht als Tun, hebt jedoch besonders die Zitatförmigkeit des doing gender und dessen machtvollen Antrieb durch normative Vorgaben heterosexueller Zweigeschlechtlichkeit hervor. Auch hier werden Geschlechterunterschiede nicht einfach reproduzierend festgestellt, sondern in deren gesellschaftlich-kulturell vermittelter Machtförmigkeit wie in deren Kontingenz und Beweglichkeit als ,Improvisationen im Rahmen des Zwangs' (Butler 2009:9) nachvollziehbar gemacht (vgl. bspw. Fritzsche 2003; Tervooren 2006; Fritzsche/Tervooren 2006; Hartmann 2012). Konzeptuell zielen dekonstruktive Perspektiven in der pädagogischen Praxis darauf, die real gelebte Vielfältigkeit an geschlechtlichen und sexuellen Lebensweisen als ein Phänomen gesellschaftlicher Pluralisierungs- wie 
Norm(alis)ierungsprozesse aufzugreifen und dabei in einer genderreflexiven Weise neben Cis-Geschlechlichkeit und Heterosexualität auch LGBTIQ*+Lebensweisen als selbstverständlich aufzugreifen. Genderreflexivität bedeutet dabei den normativen Zusammenhang von sex (anatomisches Geschlecht), gender (soziales Geschlecht) und desire (sexuelles Begehren) kritisch-dekonstruktiv zu reflektieren (vgl. bspw. Hartmann 2014, 2017b; Huch/Lücke 2015; Schmidt et al. 2015; Busche et al. 2018).

Last but not least führt auch die Debatte um Intersektionalität und interdependente Kategorien beim Thema geschlechterreflektierter Professionalität und Professionalisierung zu einem Einbeziehen weiterer Differenzkategorien (vgl. bspw. Lutz/Wenning 2001; Diehm/Messerschmidt 2013; Walgenbach 2014; Budde et al. 2016). Hier geht es insbesondere darum, das vielschichtige und wechselseitige Bedingt- wie Verwobensein der bislang getrennt gedachten Kategorien zu untersuchen sowie damit zusammenhängende Diskriminierungsmechanismen und die darunterliegenden Machtverhältnisse aufzudecken. Intersektionale Ansätze in der Erziehungswissenschaft diskutieren Differenzen zwar durchgehend macht- und normenkritisch, noch selten finden sich in der deutschsprachigen Debatte jedoch auch poststrukturalistisch-dekonstruktive „Infragestellungen von scheinbar eindeutigen Identitäten, Geschlechtern und Körpern und deren Kontingenz" (Tuider 2012: 83).

\section{Aktuelle Professionalisierungstheorien und -debatten - explizit geschlechtsneutral und implizit gegendert?}

In Professionalisierungstheorien selbst sind zunächst weder Geschlecht noch Vergeschlechtlichung explizit aufgegriffen. Mit Blick auf Professionalität und Professionalisierung in (und für) pädagogische(n) Handlungsfelder(n) werden hauptsächlich drei theoretische Ansätze (kontrovers) diskutiert.

1. Kompetenztheoretische Ansätze gehen davon aus, dass Professionalität im Zusammenspiel von professionellem Wissen (pädagogisches Wissen, Fachwissen und fachdidaktisches Wissen) und affektiv-motivationalen Charakteristika (fachbezogene und persönliche Überzeugungen, Berufsmotivation) sowie von Organisations- und Beratungswissen erfasst und entwickelt werden kann (vgl. bspw. Baumert/Kunter 2006).

2. Strukturtheoretische Ansätze verweisen eher auf die Ungewissheit pädagogisch verantworteter Entscheidungen in offenen Situationen oder auf 
die Antinomien, denen Pädagog_innen ausgesetzt sind und die diese reflektieren, moderieren und bewältigen müssen (vgl. bspw. Helsper 1996; Oevermann 1996; Helsper/Tippelt 2011).

3. Berufsbiographisch orientierte Ansätze folgen einem sich lebensgeschichtlich über verschiedene Phasen entwickelnden und mit Kontinuitäten und Brüchen verbundenen Verständnis von Professionalität, das eine berufsbiographische Entwicklungsherausforderung darstellt (vgl. bspw. Terhart 2011).

Inwiefern in geschlechtsneutral argumentierenden Debatten überkommene Implikationen von Geschlecht weiterhin eine nicht zu unterschätzende - wenngleich zumeist nicht reflektierte - Rolle spielen, lässt sich am Beispiel der Debatte um die Professionalisierung Sozialer Arbeit und der Frage, inwiefern diese eine Profession ist, aufzeigen. Heite (2009b: 50) geht davon aus, dass Geschlecht und die Wissensbestände zu geschlechtsbezogenen Eigenschaften konstitutiv für den Status Sozialer Arbeit sind. So zeichnen Bereswill und Ehlert (2012: 98f.) bspw. nach, wie innerhalb der Professionalisierungsdebatte die Tätigkeiten Sozialer Arbeit abgewertet und abgewehrt werden, die beziehungsorientiert mit Weiblichkeit assoziiert sind, und wie diese durch neutralisierte Werte überwunden werden sollen. Sie bezeichnen es als ein Paradox, dass die Akteur_innen der Professionsdebatte sich dabei für die Anerkennung einer Profession einsetzen, die mehrheitlich von Frauen ausgeführt wird, ohne die Bedeutung von Geschlechterkonstruktionen und -verhältnissen zu thematisieren. Sie erkennen darin einen androzentrischen bias und „Versuche eines undoing gender" (ebd.: 101), die - an bürgerlichen Idealen des autonomen Individuums orientiert - die beziehungsförmigen Anteile der Sozialen Arbeit weg von diffusen, kaum kontrollierbaren Dynamiken in der Hilfebeziehung hin zu ,einer allseits reflexionsfähigen Handlungskompetenz“ (ebd.) betreiben und Geschlechtsneutralität dabei jedoch nur vordergründig herstellen können.

\section{Aktuelle Korrespondenzen von Geschlechter- und Professionalisierungsdiskurs}

Auch in der Debatte zu geschlechterreflektierter Professionalität lassen sich verschiedene professionalisierungstheoretische Zugänge ausmachen. Die Betonung der Wichtigkeit, eine auf Wissen-Können-Wollen aufbauende Genderkompetenz zu entwickeln (vgl. Metz-Göckel/Roloff 2002; Kunert-Zier 2005), korrespondiert mit kompetenztheoretischen Professionalisierungstheorien. Verschiedene Studien können nachweisen, wie Genderkompetenz, basierend auf einer Dekonstruktion vergeschlechtlichter pädagogischer Praktiken und 
der Reflexion der eigenen (auch biografischen) Verwobenheit in gesellschaftlich virulente Systeme von Binarität, Differenzkonstruktion und Stereotypisierung, eine notwendige Voraussetzung für professionelles Handeln im pädagogischen Feld darstellt. Genderkompetenz - auf sozial-konstruktivistischer Ebene als reflektierte Dramatisierung, Entdramatiserung und/oder Nicht-Dramatisierung von Geschlecht verstanden - wird als ein wichtiges Kriterium pädagogischer Professionalität beschrieben (bspw. bei Baar 2010; Budde/Venth 2010; Schneider 2013). Aufgrund seines individualisierenden und Machbarkeit suggerierenden Fokus steht eine Konzentration auf Genderkompetenz in jüngster Zeit jedoch auch in der Kritik. Im Zuge einer Hinterfragung neoliberaler Haltungen sowie eines technologischen Verständnisses von Kompetenz, das Widersprüche, Antinomien sowie intersektionale Zusammenhänge ausblendet, wird demgegenüber eine diese Aspekte ebenso berücksichtigende ,Gender-Kompetenzlosigkeitskompetenz' eingefordert (vgl. Kleiner/Klenk 2017).

Korrespondenzen zwischen Gendertheorien und Professionalisierungstheorien bestehen weiterhin dort, wo ein zentrales Moment von pädagogisch-professionellem Handeln darin festgemacht wird, genderbezogene Spannungsverhältnisse zu reflektieren und zu moderieren (vgl. Hartmann 2018: 48). Hier geht es bspw. um die Paradoxie, Kategorien überwinden zu wollen und sie doch aufrufen zu müssen, um dekonstruierend mit ihnen arbeiten zu können, oder um den Konflikt zwischen einer anerkennenden ,,performativen Erzeugung von Differenz und der Besonderung“" derselben (Riegel 2016: 112). Ein weiteres Spannungsverhältnis liegt darin, dass es mit Blick auf Geschlechterdifferenzen nicht nur um Anerkennung, sondern auch um Verteilungsgerechtigkeit geht. Die Paradoxien sind strukturell verankert und „durch individuelles Agieren und pädagogisches Handeln allein nicht aufzulösen“(ebd.: 113). Auch besteht die Herausforderung, zum einen die Zerrissenheit zwischen vorherrschenden identitätsorientierten Imperativen zu kohärenten Geschlechtsidentitäten und den innerpsychischen Ambivalenzen und Verlustspuren der Individuen zu balancieren (vgl. Hartmann 2002: 156ff.) und zum anderen mit dem Spannungsverhältnis, das zwischen geschlechtlicher und sexueller Identität auf der einen und einem Recht auf geschlechtliche und sexuelle Nicht-Identifizierung auf der anderen Seite aufgespannt ist, umzugehen (vgl. Offen 2017: 127). Ein weiteres Paradox mag zwischen dem einfachen Ansprechen einer geschlechtlichen und sexuellen Vielfalt liegen, das ohne macht- und identitätskritische Perspektiven Gefahr läuft, vorherrschende Hierarchisierungen entgegen besserer Absicht zu reproduzieren, und einer heteronormativitätskritisch und dekonstruktiv ausgerichteten Pädagogik, die vielfältige geschlechtliche und sexuelle Lebensweisen entsprechend reflektiert (vgl. Hartmann 2014).

Korrespondenzen $\mathrm{zu}$ berufsbiografischen Professionalisierungstheorien zeigen sich beispielsweise in Untersuchungen, die Geschlecht als strukturierende Kategorie in den beruflichen Orientierungen nachweisen. So beschreibt 
Bobeth-Neumann (2013), wie sich unterschiedliche Ermutigungsstrukturen gegenüber Frauen und Männern sowie die Ko-Konstruktion von Führung und Männlichkeit im Feld Grundschule auf den Karrierewunsch ,Schulleitung ‘ auswirken. Buchen (1991) ermittelt unterschiedliche Orientierungen von Gesamtschullehrer_innen vor allem in Bezug auf die Betonung der Beziehung zu den Schüler_innen (durch Lehrerinnen) sowie die Fokussierung auf fachliche Inhalte (durch Lehrer). Damit bestätigt sie die Ergebnisse von Flaake (1988), die männlichen Sekundarstufenlehrer die Orientierung an der professionellen Identität des Wissensvermittlers zuschreibt, während (zumindest jüngere) Lehrerinnen eher die Beziehungsebene zu den Schüler_innen fokussieren. Düro (2008) kann in ihrer Studie ein solchermaßen differentes Professionsverständnis nicht erkennen; dennoch identifiziert sie Geschlechterreviere, hinter denen „eine grundsätzliche Konkurrenz im Wettstreit um Macht und Einfluss zu erkennen" (ebd.: 234) sei. Während im Vorangegangenen Lehrer_innen im Fokus stehen, geht es in den folgenden Studien um das berufliche Selbstverständnis von Mädchen- und Jungenarbeiter_innen: Schütz (2016) zeigt auf, wie durch die Reflexion der eigenen Berufsbiografie im Rahmen eines Interviews den befragten Sozialpädagogen ihre eigene Professionalität als Jungenarbeiter - sie nennen als wichtigste Merkmale Reflexionsvermögen, Sensibilität und Authentizität - erst bewusst wird und ihnen zugleich Erfolge professionellen Handels schwer messbar erscheinen - ebenso wie die Unterscheidung zwischen professionell und unbewusst persönlich motiviertem Handeln. Transformationen von der DDR in ein wiedervereinigtes Deutschland in Verbindung mit Profession, Biografie und Geschlecht beleuchtet Daigler (2008) und stellt heraus, wie sich das berufliche Selbstverständnis von Sozialpädagoginnen in ostdeutschen Bundesländern zwischen der Bewahrung von Kontinuität - zum Beispiel beim Anknüpfen an Gleichberechtigungsvorstellungen in der Mädchenarbeit vor und nach der Wende - oder über Entpolitisierung durch eine Konzentration auf das Familiäre, Vertraute entfaltet.

\section{Paradigmen, Bildungsorte und Vermittlungsfragen - Transformationen im Spannungsverhältnis von Theorie, Politik und Praxis}

Die aufgezeigten theoretischen und konzeptionellen Ansätze für eine geschlechterreflektierte Professionalisierung bieten unterschiedliche Zugänge in den jeweils ,zuständigen" Bildungsbereichen Politik, Wissenschaft und (Aus)Bildung. Hier fällt auf, dass sich mit der seit Ende der 1990er Jahre einsetzenden sogenannten ,empirischen Wende' vor allem in der Bildungspolitik, 
aber (zumindest teilweise) auch in der Bildungsforschung (vgl. BuchhaasBirkholz 2009; Fend 2010) sowie in den (Aus)Bildungsstätten begrifflich das Kompetenzparadigma durchgesetzt zu haben scheint. Nicht nur die Bildungspläne der Länder für Kitas sowie für Schulen folgen kompetenzorientierten Zugängen. Auch im Rahmen der Standards für die Lehrer_innenbildung (vgl. KMK 2004/2014) wird Kompetenz großgeschrieben. Ebenso hat der Fachbereichstag Soziale Arbeit mit dem ,Qualifikationsrahmen Sozialer Arbeit' als anerkannte Referenzgrundlage für die Studiengänge Sozialer Arbeit seit mehr als 10 Jahren eine Kompetenzorientierung mittels definierter Deskriptoren eingeführt (vgl. Fachbereichstag Soziale Arbeit 2016).

Dass der strukturtheoretische wie der biografieorientierte Professionalisierungsansatz nicht so prominent im Diskurs um die wissenschaftliche Ausbildung pädagogischer Fachkräfte angekommen zu sein scheint, stimmt nachdenklich und wirft einige Fragen auf: Werden deren Erkenntnis- und Qualifizierungspotentiale unter dem Kompetenzbegriff eher subsumiert oder marginalisiert? Inwiefern könnte der Boom des Kompetenzbegriffs mit dem bildungspolitischen Paradigma der Steuerung des Bildungssystems auf der Grundlage groß angelegter, (international) vergleichender quantitativer Studien und seiner einfacheren Operationalisierung zusammenhängen? Welche Rollen nehmen dabei universitäre wie außeruniversitäre Bildungsforschung, welche die Erziehungswissenschaft als deren Leitdisziplin ein? Wie ist es zu verstehen, dass das Kompetenzparadigma gerade von außeruniversitären Forschungseinrichtungen und Wirtschaftsorganisationen (wie bspw. der MaxPlanck-Gesellschaft, der OECD etc.) propagiert und vorangetrieben wurde (und nach wie vor wird), diese an den Professionalisierungsprozessen von (angehenden) Lehr- und pädagogischen Fachkräften aber überhaupt nicht beteiligt sind?

Hier schließt sich die Frage danach an, was konkret an den Orten passiert, an denen Professionalisierungsprozesse angebahnt werden und stattfinden (sollten). Dies auch angesichts der einleitend beschriebenen, immer wieder beklagten Kluft zwischen erziehungswissenschaftlichen Erkenntnissen und fachlichem Handeln. Was bedeutet es, wenn schon dem breit rezipierten Modell der ,reflexiven Koedukation" eine weitgehend fehlende Umsetzung in der Schule attestiert werden muss (vgl. Kampshoff/Scholand 2017)? Wieso werden die hier entwickelten Theorien und Konzepte so selten als Reflexionsfolie und Inspirationsquelle genutzt? Wieso finden darüber hinaus poststrukturalistisch bzw. heteronormativitätskritisch und intersektional inspirierte Ansätze in den Debatten um Schule und Geschlecht viel seltener Eingang als sozialkonstruktivistische (vgl. Kleiner/Klenk 2017)? Hier gilt es, nicht nur das Verhältnis von Wissenschaft und Bildungspraxis zu reflektieren. Vielmehr hat auch der Umgang mit den zu konstatierenden Spannungsverhältnissen zwischen erziehungswissenschaftlichem, bildungs- und gendertheoretischem (For- 
schungs-) Wissen auf der einen und bildungspolitischen Konjunkturen, alltagstheoretischen Ausrichtungen und berufspraktischen Erkenntnissen auf der anderen Seite eine größere Aufmerksamkeit verdient.

Eine wichtige mit Forschung verbundene Aufgabe ist Vermittlung. Eine gängige und zweifelsohne zutreffende These ist die, dass der Vermittlung von wissenschaftlichen Debatten in die Praxis, in deren Diskurse, Konzepte und Alltagspraxen z. B. über Fort- und Weiterbildung, insbesondere aber über die Ausbildung sorgfältiger Rechnung getragen werden muss. Eine weitere, nicht minder überzeugende These ist die, dass ein Bewusstsein für die Bedeutung geschlechter- bzw. genderreflektierender Professionalität bedeuten müsste, mehr Zeiten und Räume für den damit verbundenen kontinuierlichen Professionalisierungsprozess zu schaffen. Denn wie bereits festgestellt: Geschlechterreflektierte Professionalität ist wesentlich mehr als auf dem aktuellen Stand der Gender-Debatten zu sein. Geht es damit verbunden um Reflexion, Haltung, Habitus, um eine bestimmte Qualität von Wissen, Können und Wollen, um ein Balancieren von Aporien und eine reflexive Bewusstheit von lebensgeschichtlichen Bezügen zum eigenen beruflichen Handeln, dann liegen zentrale Bedingungsfaktoren von Professionalität in angemessenen Zeiten und Räumen für die unumgänglichen Reflexionsprozesse begründet.

Basis für diese Reflexion ist Wissen, das ermöglicht, ,zu wissen, was man tut" (Dewe 2009: 56). Zugleich ist hier eben nicht nur wissenschaftliches Wissen gefragt, sondern auch praktisches Handlungswissen, das von Praktiker_innen auch im Austausch mit Kolleg innen und außenstehenden Berater innen konkretisiert wird und ständig zu überprüfen ist (vgl. Heiner 2007: 185). Professionalisierungsprozesse benötigen entsprechend ausreichende Selbstvergewisserungsinstanzen der Professionalisierung, wie sie bspw. in Studienprojekten, in kollegialer Beratung und Supervisionsgruppen oder im Rahmen von Praxisforschungsprojekten gegeben sind. Das beispielsweise. in Praxisforschungsprojekten umsetzbare Format der Reflecting Group (vgl. Wigger et al. 2012: 253; Busche/Hartmann 2018: 76; Busche/Streib- Brzič in diesem Band) referiert auf ein Verständnis von Professionalisierung, in dessen Prozess sich wissenschaftliches und berufspraktisches Handlungswissen begegnen und eine Relationierung beider Wissensarten stattfindet. So werden wissenschaftliche Erkenntnisse nicht einfach transferiert, sondern vielmehr in der Begegnung mit beruflichem Erfahrungswissen transformiert. Denn es ist die konzeptuelle Übertragung auf die spezifische Situation der jeweiligen pädagogischen Praxis mit der dabei stattfindenden, das eigene Handlungswissen heranziehenden Verschiebung, die professionelles Handeln erst ermöglicht. Reflecting Groups bieten, dem Gedanken einer dialogischen Verständigung folgend, Fachkräften aus Wissenschaft und Bildungspraxis die Möglichkeit, die unterschiedlichen Erfahrungs- und Wissenslogiken der verschiedenen Felder zu berücksichtigen und einer hierarchischen, Transfer-Mentalität' durch eine Relationierung von 
Wissensbeständen in einem ko-produktiven Vorgehen zu begegnen. Entsprechende Zugänge sind umso mehr gefragt, je stärker genderreflektierende Professionalisierung als ein kollektiver Prozess diskursiver Entwicklung und die Logik genderreflektierten professionellen Handelns in der Balancierung von Spannungsverhältnissen gesehen wird.

\section{$8 \mathrm{Zu}$ den Schwerpunkt-Beiträgen in diesem Band}

Wir freuen uns, mit dem vorliegenden Jahrbuch eine ganze Reihe von empirischen Studien zu geschlechterreflektierter Professionalisierung und Professionalität in den Bereichen Kita, Schule, außerschulische Jugendarbeit, Lehramtsstudium bzw. in der Elementar- und Sexualpädagogik sowie der Jugendmuseumsarbeit präsentieren zu können und danken den Autor_innen für ihre die Debatte bereichernden Beiträge.

Die ersten Beiträge wenden sich der Bearbeitung von vielfältigen Lebensweisen im Fokus von Professionalität zu. Im Zentrum des Artikels von Florian Cristobal Klenk Interdependente Geschlechtervielfalt als un/be/deutende Anforderung an pädagogische Professionalität stehen Überlegungen zum Verhältnis von Kontingenz, Differenz und pädagogischer Professionalität. Nach einem Überblick zum (internationalen) Forschungsstand zur Thematisierung vielfältiger geschlechtlicher und sexueller Lebensweisen in der Schulpädagogik und sich daraus ergebender Entwicklungs- und Forschungsdesiderate für eine geschlechterreflektierende Professionalität und Professionalisierung, gibt Klenk Einblick in ein größeres Forschungsvorhaben zu Erfahrungen von Lehrer_innen im schulischen Handeln mit und bei der Thematisierung vielfältiger Lebensweisen entsprechend derer Selbstaussagen. Konkret diskutiert Florian Cristobal Klenk, wie Lehrpersonen einer beruflichen Schule vor dem Hintergrund vielfältiger geschlechtlicher und sexueller Lebensweisen mit Irritationen und auftretenden Handlungsungewissheiten umgehen, welche Funktion den in episodischen Interviews sichtbar werdenden situations- und differenzbezogenen Deutungen aus Perspektive der Lehrkräfte für professionelles pädagogisches Handeln zukommt sowie welche impliziten Normen dabei zu Tage treten. Dabei zeigt sich, wie Heterosexualität als selbstverständlicher Teil des Schulalltags wahrgenommen und Homosexualität demgegenüber als marginalisierte und fragile Subjektposition verhandelt wird, die als durch Jungen mit Migrationshintergrund gefährdet erscheint. Während Trans* als ,das Andere der schulischen Normalität angetroffen wird, finden sich die Lehrpersonen zwischen Zugzwängen und Vermittlungschancen wider. In Berücksichtigung der Verschränkungen von Trans*-Geschlechtlichkeit und Dis/Ability kann 
Klenk entlang der geschilderten Fallgeschichte zwei verschiedene Bearbeitungsmodi im Umgang mit interdependenter Geschlechtervielfalt rekonstruieren. Dabei analysiert er un/be/deutende Wechselwirkungen von differenzbezogener Wahrnehmung und Orientierung der Lehrpersonen mit (multi)professionellen Wissens- und schulischen Verhaltensordnungen.

Mart Busche und Uli Streib-Brzič beschäftigen sich in ihrem Beitrag Die Entwicklung heteronormativitätskritischer Professionalität in ReflexionsWorkshops - Zur Verbindung von pädagogischem Erfahrungswissen und wissenschaftlichem Erkenntniswissen im Kontext von Praxisforschung mit dem Potential des Rückspiegelns empirisch generierten Wissens in die Praxis der Jugendbildungsarbeit. Die Autor_innen gehören dem Team des Praxisforschungsprojekts, VieL*Bar: Vielfältige geschlechtliche und sexuelle Lebensweisen in der Bildungsarbeit - Didaktische Potentiale und Herausforderungen museumspädagogischer Zugänge' an, das die museumspädagogische Arbeit des Modellprojekts ,All Included! - Museum und Schule gemeinsam für geschlechtliche und sexuelle Vielfalt ' untersucht hat. In wissenschaftliches Wissen und berufliches Erfahrungswissen zusammenführenden Reflexions-Workshops würdigten Praktiker_innen und Wissenschaftler_innen gemeinsam Bedingungen für ein Gelingen praktischer Arbeit zum Themenfeld bzw. eruierten Herausforderungen für diese. Im Feld der heteronormativitätskritischen Bildungsarbeit spielt dabei auch das Wissen der Communities, in denen vielfältige geschlechtliche und sexuelle Lebensweisen gelebt werden, eine große Rolle. Im Beitrag werden zwei Diskussionssequenzen zum Thema ,Trans** und zum Thema ,Ziele heteronormativitätskritischer Bildungsarbeit' analysiert und es wird herausgearbeitet, wie Professionalisierung durch eine interdisziplinäre Reflexionsarbeit im noch jungen Feld dieser pädagogischen Arbeit vorangetrieben werden kann.

Gleichheitsrhetorik und Ungleichheitsverhältnisse zu geschlechtlicher und sexueller Vielfalt als paradoxales Spannungsfeld der Schule begreifend, das professionellen Handelns bedarf, fragen Carolin Vierneisel und Johannes Nitschke nach der Bedeutung dieses Themenfelds in der Ausbildung von Lehrkräften. In ihrem Beitrag (De-)Professionalisierungstendenzen?! Vielfalts*sensible Bildung im Lehramtsstudium stehen neben (inter-)nationalen Forschungsergebnissen Erkenntnisse aus einer quantitativen Erhebung zu sexueller und geschlechtlicher Vielfalt in der Lehre der Universität Leipzig im Mittelpunkt. Hier sinkt die Unterstützung der Thematisierung von Vielfalt* in den Angaben der befragten Lehrenden kontinuierlich, je konkreter der Bezug zu der eigenen Lebens- bzw. Arbeitswelt und damit zum eigenen Handlungsund Aufgabenrepertoire gegeben ist. Die Diskrepanz zwischen der zugemessenen Bedeutung von Vielfalt* und dem selbst attestierten Wissen darum einerseits und der nicht gleichermaßen erfolgenden Berücksichtigung in der Lehrpraxis andererseits interpretieren die Autor innen als eine Tendenz zur pädagogischen De-Professionalisierung: Die Universität selbst erscheint als 
ein Raum paradoxer Pluralisierungen, in dem es den Lehrenden nicht in angemessener Weise gelingt, relevantes Wissen der erziehungswissenschaftlichen Geschlechterforschung im Lehramtsstudium zu vermitteln.

Im folgenden Kapitel fokussieren zwei Beiträge geschlechterreflektierte Professionalität im Elementarbereich. Einen Beitrag für eine geschlechterreflektierende Professionalisierungsdebatte jenseits von Wirksamkeitsanforderungen leistet Melanie Kubandt in ihrem Aufsatz Ansprüche an ein geschlechtergerechtes, professionelles Handeln im Elementarbereich - Ethnographische Perspektiven auf Ungewissheiten, Komplexitäten und Grenzen im pädagogischen Alltag. Am Beispiel des Anspruchs einer Realisierung von Geschlechtergerechtigkeit in Kindertageseinrichtungen lotet sie Chancen und Grenzen einer ethnographischen Forschungsstrategie mit Blick auf doing gender aus. Zunächst problematisiert sie, was genau unter Geschlechtergerechtigkeit zu verstehen ist und dass diese je nach inhaltlicher Ausrichtung politischer Vorgaben oder wissenschaftlicher Studiendesigns sehr unterschiedlich gedacht werden kann, sich die verschiedenen Verständnisse sogar widersprechen können. Dies führt unter Umständen - wie die Autorin anhand von Praxisbeispielen aufzeigt - zu Unsicherheiten und nebulösen Flecken im pädagogischen Alltag. Kubandt erweitert normativ geprägte Sichtweisen auf dieses Thema, wie sie etwa in Bildungsplänen formuliert sind, durch Aufzeigen von Praktiken im Kontext eines vermeintlich geschlechtersensiblen Handelns. In einer deskriptiven Perspektive wird nachgezeichnet, wie Praktiken im Elementarbereich sich gestalten mit dem Ziel, erst einmal zu sehen, , wie sie tun, was sie tun'. Auslassungen seitens der Fachkräfte im Hinblick auf ihren unbewussten Umgang mit der Geschlechterthematik und auch Ungewissheiten im pädagogischen Alltag lassen sich auf diese Weise aufzeigen.

Susann Fegter, Anna Hontschik, Eszter Kadar, Kim-Patrick Sabla und Maxine Saborowski konzipieren professionelles Handeln wie Geschlecht als diskursive Konstruktion von Herausforderungen, praktischen Handlungsproblemen und legitimen Strategien des Umgangs im beruflichen Feld. In ihrem Beitrag Bezüge auf Familie als Moment der Vergeschlechtlichung pädagogischer Professionalität: Diskursanalytische Perspektiven auf Äußerungen in Gruppendiskussionen mit Kita-Teams stellen sie die Frage, wie pädagogische Fachkräfte in Kindertageseinrichtungen das Verhältnis von Professionalität und Geschlecht im Austausch über das eigene berufliche Handeln herstellen und ordnen. Die Autor_innen analysieren hierzu beispielhaft zwei Auszüge aus im Rahmen eines Forschungsprojekts durchgeführten Gruppendiskussionen, in denen die Themenfeldern Kooperation mit Kolleg_innen, Disziplinierung sowie Bedarf an Männern in Kitas von den Fachkräften bearbeitet werden. Das Autor_innenteam rekonstruiert diskursanalytisch, wie die Diskussionsteilnehmer_innen das professionelle Handeln vergeschlechtlichen, indem dieses auf eine binäre, komplementäre und heteronormative Familien(geschlechter)ordnung bezogen wird. Das historische Konzept der ,geistigen Mütterlichkeit ‘ 
scheint dabei abgelöst von jenem einer, Väterlichkeit', das vor allem über einen Rekurs auf Erfahrung in Anschlag gebracht wird.

Herausforderungen sexualpädagogischer Professionalisierung stehen im Mittelpunkt der folgenden beiden Aufsätze. Ausgangspunkt des Beitrags von Anja Eichhorn mit dem Titel Doing Sexual Agency: Sexuelle Handlungsfähigkeit sexuell missbrauchter jugendlicher Mädchen in der stationären Jugendhilfe ist die Erkenntnis, dass Mädchen, die sexualisierte Gewalt erfahren haben, im Kontext von Jugendhilfe eher mit Maßnahmen zu Gewaltprävention begleitet werden denn mit Angeboten, in denen auch lustbetonte Seiten der Sexualität eine Rolle spielen. Die Autorin nimmt dies zum Anlass für eine grundlegende Diskussion von Begriffen, die in der diesbezüglichen pädagogischen Arbeit mit Mädchen, verwendet werden. Auf einer Metaebene werden Überlegungen angestellt, wie schon auf dieser Ebene in der Jugendhilfe sexueller Reviktimisierung vorgebeugt und auch sexuelle Selbstbestimmtheit unterstützt werden könnte. Dabei greift die Autorin das Konzept der sexual agency auf, das sich in diesem Kontext anbietet für individuelle Handlungs- und Gestaltungsspielräume einer gelingenden Jugendsexualität. Dies bezieht u.a. auch den selbst gewählten Verzicht des Auslebens von Sexualität mit ein. Die traumatischen Erfahrungen der Mädchen, die hier im Zentrum stehen, gilt es bei sexual agency in vielfacher Hinsicht zu berücksichtigen. Zum Beispiel müssten sexuelle Handlungszwänge stärker als bislang miteinbezogen werden, um zu verhindern, dass ein quasi neoliberales Ideal eines autonomen, emanzipierten, aktiv begehrenden Individuums die jungen Frauen unter Druck setzt. Wird hingegen sexual agency als performativer Akt betrachtet, weitet sich dieses in Richtung eines doing sexual agency aus und bietet somit neuartige Anschlüsse für pädagogische Konzepte in der Jugendhilfe.

Mit ihrem Beitrag Geschlechterreflektierte sexuelle Bildung? Heteronormativität und Verletzbarkeit als Herausforderungen sexualpädagogischer Professionalisierung analysiert Marion Thuswald sexualpädagogische Aus-, Fort- und Weiterbildungsformate in Österreich. Auf Grundlage einer ethnografischen Studie, bei der einführende sexualpädagogische Bildungsveranstaltungen für (angehende) Lehrer_innen und andere pädagogisch Tätige beobachtet wurden, werden drei verschiedene Verständnisse von Sexualität dargestellt, die nur zum Teil als geschlechterreflektiert gelten können und nur wenig den menschenrechtsbasierten und der Gleichstellung der Geschlechter verpflichteten Leitlinien der UNESCO zu einer comprehensive sexuality education entsprechen. Nur das identifizierte Verständnis von Sexualität als Wahrnehmung von Wollen, Anziehung und Erregung mache geschlechtliche, sexuelle und amouröser Vielfalt besprechbar und biete Anschlussmöglichkeiten auch für gewaltpräventive Arbeit. Thuswald betont den Zusammenhang zwischen Heteronormativität, Verletzbarkeit und sexueller Gewalt und kommt vor dem Hintergrund ihrer Untersuchungsergebnisse zu dem Schluss, dass die Entwicklung und Etablierung nicht-heteronormativer Sexualitätsverständnisse sowie 
geschlechterreflektierter sexualpädagogischer Ansätze eine zentrale Herausforderung gegenwärtiger sexualpädagogischer Professionalisierung darstellt.

\section{Literaturverzeichnis}

Baader, Meike Sophia (2018): Von der Normalisierung zur De-Zentrierung nach 1968. Mütterlichkeit, Weiblichkeit und Care in der Alten und in der Neuen Frauenbewegung. In: Langer, Antje/Mahs, Claudia/Rendtorff, Barbara (Hrsg.): Weiblichkeit. Ansätze und Theoretisierung. Opladen: Barbara Budrich, S. 15-37.

Baar, Robert/Schönknecht, Gudrun (2018): Außerschulische Lernorte: didaktische und methodische Grundlagen. Weinheim/Basel: Beltz.

Baar, Robert (2014): Gender Studies in der Erziehungswissenschaft: Aspekte einer gendersensiblen (Grund-) Schulpädagogik. In: Fellner, Astrid M./Conrad, Anne/Moos, Jennifer J* (Hrsg.): Gender überall!? Beiträge zur interdisziplinären Geschlechterforschung. St. Ingbert: Röhrig, S. 145-162.

Baar, Robert (2010): Allein unter Frauen. Der berufliche Habitus männlicher Grundschullehrer. Wiesbaden: VS. https://doi.org/10.1007/978-3-531-92408-3

Baar, Robert (2009): Feminisierung des Grundschullehrerberufs. In: Barnitzky, Horst/Grügelmann, Hans/Hecker, Ulrich/Heinzel, Frederike/Schönknecht, Gudrun/Speck-Hamdan, Angelika (Hrsg.): Kursbuch Grundschule. Frankfurt a.M.: Grundschulverband, S. 356-359.

Baumert Jürgen/Kunter, Mareike (2006) Stichwort: Professionelle Kompetenz von Lehrkräften. In: Zeitschrift für Erziehungswissenschaft 9, 4, S.469-520.

Bereswill, Mechthild (2016): Hat Soziale Arbeit ein Geschlecht? In: Deutscher Verein für öffentliche und private Fürsorge e.V. (Hrsg.): Soziale Arbeit kontrovers 16. Freiburg i. Br.: Lambertus.

Bereswill, Mechthild/Ehlert, Gudrun (2012): Frauenberuf oder (Male)Profession? Zum Verhältnis von Profession und Geschlecht in der Sozialen Arbeit. In: Bütow, Birgit/Munsch, Chantal (Hrsg.): Soziale Arbeit und Geschlecht. Herausforderungen jenseits von Universalisierung und Essentialisierung. Münster: Westfälisches Dampfboot, S. 92-107.

Bitzan, Maria (2004): Gender in der Kinder- und Jugendhilfe. In: Glaser, Edith/ Klika Dorle/Prengel, Annedore (Hrsg.): Handbuch Gender und Erziehungswissenschaft. Bad Heilbrunn: Klinkhardt, S. 461-476.

Bitzan, Maria/Daigler, Claudia (2004): Eigensinn und Einmischung. Einführung in Grundlagen und Perspektiven parteilicher Mädchenarbeit. 2. Auflage. Weinheim: Juventa.

Bobeth-Neumann, Wiebke (2013): Karriere „Grundschulleitung“. Über den Einfluss des Geschlechts beim beruflichen Aufstieg ins Schulleitungsamt. Bielefeld: transcript.

Breidenstein Georg/Kelle, Helga (1998): Geschlechteralltag in der Schulklasse. Ethnographische Studien zur Gleichaltrigenkultur. Weinheim: Juventa. 
Buchen, Sylvia (1991): Ich bin immer ansprechbar. Gesamtschulpädagogik und Weiblichkeit. Eine sozialpsychologische Frauenstudie, Weinheim: Deutscher Studienverlag. https://doi.org/10.1007/978-3-531-19345-8_7

Buchhaas-Birkholz, Dorothee (2009): Die „empirische Wende“ in der Bildungspolitik und in der Bildungsforschung. Zum Paradigmenwechsel des BMBF im Bereich der Forschungsförderung. In: Erziehungswissenschaft 20, 39, S. 27-33.

Budde, Jürgen/Offen, Susanne/Tervooren, Anja (Hrsg.) (2016): Das Geschlecht der Inklusion. Jahrbuch Frauen- und Geschlechterforschung in der Erziehungswissenschaft H. 12/2016. Opladen: Barbara Budrich

Budde, Jürgen/Debus, Katharina/Krüger, Stefanie (2011): „Ich denk nicht, dass meine Jungs einen typischen Mädchenberuf ergreifen würden." Intersektionale Perspektiven auf Fremd- und Selbstrepräsentationen von Jungen in der Jungenarbeit. In: Gender - Zeitschrift für Geschlecht, Kultur und Gesellschaft 3, 3, S.119-127.

Budde, Jürgen/Venth, Angela (2010): Genderkompetenz für lebenslanges Lernen. Bildungsprozesse geschlechterorientiert gestalten. Bielefeld: WBV.

Budde, Jürgen/Scholand, Barbara/Faulstich-Wieland, Hannelore (2008): Geschlechtergerechtigkeit in der Schule. Eine Studie zu Chancen, Blockaden und Perspektiven einer gender-sensiblen Schulkultur. Weinheim: Juventa.

Busche, Mart/Hartmann, Jutta/Nettke, Tobias/Streib-Brciz, Uli (2018): Heteronormativitätskritische Jugendbildung. Reflexionen am Beispiel eines museumspädagogischen Modellprojekts. Bielefeld: transcript.

Busche, Mart/Hartmann, Jutta (2018): Multiperspektivisch \& partizipativ - Zum Forschungsdesign des Praxisforschungsprojekts VieL*Bar. In: Busche, Mart/Hartmann, Jutta/Nettke, Tobias/Streib-Brciz, Uli (Hrsg.): Heteronormativitätskritische Jugendbildung. Reflexionen am Beispiel eines museumspädagogischen Modellprojekts. Bielefeld: transcript, S. 69-81. https://doi.org/10.14361/978383944 2418004

Busche, Mart/Cremers, Michael (2012): Jungenarbeit und Intersektionalität. Online unter: http://portal-intersektionalitaet.de/theoriebildung/ueberblickstexte/ buschecremers/ [Zugriff: 18.01.2019].

Busche, Mart/Maikowski, Laura/Pohlkamp, Ines/Wesemüller, Ellen (2010): Feministische Mädchenarbeit weiterdenken. Eine Einleitung. In: Dies. (Hrsg.): Feministische Mädchenarbeit weiterdenken. Zur Aktualität einer bildungspolitischen Praxis. Bielefeld: transcript, S. 7-20. https://doi.org/10.14361/transcript.9783839413838

Butler, Judith (2009): Die Macht der Geschlechternormen und die Grenzen des Menschlichen. Frankfurt a.M.: Suhrkamp.

Campe, Joachim H. (1789/1988): Väterlicher Rath für meine Tochter. Ein Gegenstück zum Theophron. Paderborn: Schöningh.

Career Counselling for Teachers (CCT) (2018): Laufbahnberatung für Lehrerinnen und Lehrer. Online unter: http://www.cct-germany.de/de/0/pages/index/45 [Zugriff: 02.11.2018].

Connell, Robert W. (2000): The Men and the Boys. Oxford: Allen \& Unwin.

Daigler, Claudia (2008). Biografie und sozialpädagogische Profession: eine Studie zur Entwicklung beruflicher Selbstverständnisse am Beispiel der Arbeit mit Mädchen und jungen Frauen. Weinheim: Juventa.

Dausien, Bettina (2009a): Sozialisation - Biografie. Theoretische Diskurse und Forschungsperspektiven. Wiesbaden: Springer VS. 
Dausien, Bettina (2009b): Differenz und Selbst-Verortung - Die soziale Konstruktion von Geschlecht in Biographien als Forschungskonzept. In: Aulenbacher, Brigitte/Riegraf, Birgit (Hrsg.): Erkenntnis und Methode. Wiesbaden: Springer VS, S. 157-177. https://doi.org/10.1007/978-3-531-91566-1 9

Dekker, Arne (2012): Online Sex. Körperliche Subjektivierungsformen in virtuellen Räumen. Bielefeld: transcript.

Dewe, Bernd (2009): Reflexive Professionalität: Maßgabe für Wissenstransfer und Theorie-Praxis-Relationierung im Studium der Sozialarbeit. In: Riegler, Anna/Hojnik, Sylvia/Posch, Klaud (Hrsg.): Soziale Arbeit zwischen Profession und Wissenschaft. Vermittlungsmöglichkeiten in der Fachhochschulausbildung. Wiesbaden: Springer VS, S. 47-63.

Diehm, Isabell/Messerschmidt, Astrid (Hrsg.) (2013): Das Geschlecht der Migration. Bildungsprozesse in Ungleichheitsverhältnissen. Jahrbuch Frauen- und Geschlechterforschung in der Erziehungswissenschaft H. 9/2013. Opladen: Barbara Budrich

Dietze, Gabriele/Hornscheidt, Lann/Palm, Kerstin/Walgenbach, Katharina (2007): Gender als interdependente Kategorie. Neue Perspektiven auf Intersektionalität, Diversität und Heterogenität. Opladen: Barbara Budrich.

Düro, Nicole (2008): Lehrerin - Lehrer: Welche Rolle spielt das Geschlecht im Schulalltag? Opladen/Farmington Hills: Barbara Budrich.

Eggers, Maureen Maisha (2012): Diversität als neues Möglichkeitsfeld: Diversität als Motor einer Neustrukturierung im Verhältnis der (feministischen) Mädchenarbeit zur (kritischen) Jungenarbeit. In: Bütow, Birgit/Munsch, Chantal (Hrsg.): Soziale Arbeit und Geschlecht. Herausforderungen jenseits von Universalisierung und Essentialisierung. Forum Frauen- und Geschlechterforschung Band 34, Münster: Westfälisches Dampfboot, S. 229-245.

Emmerich, Marcus/Hormel, Ulrike (2013): Heterogenität - Diversity - Intersektionalität. Zur Logik sozialer Unterscheidungen in pädagogischen Semantiken der Differenz. Wiesbaden: Springer VS.

Fachbereichstag Soziale Arbeit (2016): Qualifikationsrahmen Soziale Arbeit (QR SozARb 2016). Version 6.0 unter: http://www.fbts.de/fileadmin/fbts/QR_SozArb_Version_6.0.pdf.

Faulstich-Wieland, Hannelore (2012): Quoten sind Machtinstrumente - Erziehung aber braucht Qualität. In: Hurrelmann, Klaus/Schultz, Tanjev (Hrsg.): Jungen als Bildungsverlierer. Brauchen wir eine Männerquote in Kitas und Schulen? Weinheim/Basel: Beltz Juventa, S. 144-154.

Faulstich-Wieland, Hannelore (2004): Doing Gender: konstruktivistische Beiträge. In: Glaser, Edith/Klika, Dorle/Prengel, Annedore (Hrsg.): Handbuch Gender und Erziehungswissenschaft. Bad Heilbrunn: Klinkhardt, S.175-191.

Faulstich-Wieland, Hannelore/Weber, Martina/Willems, Katharina (2004): Doing Gender im heutigen Schulalltag. Empirische Studien zur sozialen Konstruktion von Geschlecht in schulischen Interaktionen. Weinheim: Juventa.

Fegter, Susann (2012): Die Krise der Jungen in Bildung und Erziehung. Diskursive Konstruktion von Geschlecht und Männlichkeit. Wiesbaden: Springer VS. https://doi.org/10.1007/978-3-531-19132-4

Fend, Helmut (2010): Bildungsforschung von 1965 bis 2008 - ein Zeitzeugenbericht zu Fortschritten, Rückschlägen und Höhepunkten. In: Ritzi, Christian/Wiegmann, Ulrich (Hrsg.): Beobachten, Messen, Experimentieren. Beiträge zur Geschichte 
der empirischen Pädagogik/Erziehungswissenschaft. Bad Heilbrunn: Klinkhardt, S. 275-303.

Flaake, Karin (1988): Weibliche Identität und Arbeit in der Schule: Das unterschiedliche Verhältnis von Lehrerinnen und Lehrer zu ihrem Beruf. In: Giesche, Siegrid/Sachse, Dagmar (Hrsg.): Frauen verändern Lernen. Kiel: Hypatia, S. 180186.

Forster, Edgar (2004): Jungen- und Männerarbeit. In: Glaser, Edith/Klika, Dorle/ Prengel, Annedore (Hrsg.): Handbuch Gender und Erziehungswissenschaft. Bad Heilbrunn: Klinkhardt, S.477-491.

Fritzsche, Bettina (2003): Pop-Fans. Studie einer Mädchenkultur. Opladen: Leske \& Budrich

Fritzsche, Bettina/Tervooren, Anja (2006): Begehrensdynamiken in der Sozialisation. Perspektiven des Performativen. In: Dausien, Bettina/Bilden, Helga (Hrsg): Sozialisation und Geschlecht. Theoretische und methodologische Aspekte. Opladen/Farmington Hills: Barbara Budrich, S. 139-161.

Fritzsche, Bettina/Hartmann, Jutta/Schmidt, Andrea/Tervooren, Anja/ (Hrsg.) (2001): Dekonstruktive Pädagogik. Erziehungswissenschaftliche Debatten unter poststrukturalistischen Perspektiven. Opladen: Leske \& Budrich

Glücks, Elisabeth/Ottemeier-Glücks, Franz G. (1994): Geschlechtsbezogene Pädagogik. Ein Bildungskonzept zur Qualifizierung koedukativer Praxis durch parteiliche Mädchenarbeit und antisexistische Jugendarbeit. Münster: Votum.

Hark, Sabine/Villa, Paula-Irene (2015): „Eine Frage an und für unsere Zeit“. Verstörende Gender Studies und symptomatische Missverständnisse. In: Dies. (Hrsg.): Anti-Genderiumus. Sexualität und Geschlecht als Schauplätze aktueller politischer Auseinandersetzungen. Bielefeld: transcript, S. 15-39. https://doi.org/10.14361/9783839431443-002

Hartmann, Jutta (2018): Jugendbildung queer(en) - Zur Relevanz einer heteronormativitätskritischen Pädagogik. In: Busche, Mart/Hartmann, Jutta/Nettke, Tobias/Streib-Brciz, Uli (Hrsg): Heteronormativitätskritische Jugendbildung. Reflexionen am Beispiel eines museumspädagogischen Modellprojekts. Bielefeld: transcript, S. 19-48. https://doi.org/10.14361/9783839442418-002

Hartmann, Jutta (2017a): Dimensionen sexueller Diversität - queere und intersektionale Perspektiven. In: Klein, Alexandra/Tuider, Elisabeth (Hrsg.): Sexualität und Soziale Arbeit. Grundlagen Sozialer Arbeit. Baltmannsweiler: Schneider Hohengehren, S. 57-80.

Hartmann, Jutta (2017b): Perspektiven queerer Bildungsarbeit. In: Behrens, Christoph/Zittlau, Andrea (Hrsg.): Queer-Feministische Perspektiven auf Wissen(schaft). Interdisziplinäre Rostocker Gender und Queer Studies, Bd. 1, S. 158181.

Hartmann, Jutta (2014): Queere Professionalität als Haltung des Infragestellens und Dynamisierens. Zur Dekonstruktion geschlechtlicher und sexueller Identität in der Sozialen Arbeit. In: Sozialmagazin. Die Zeitschrift für Soziale Arbeit: Queerfeldein durch die Soziale Arbeit 39, 3/4, S. 22-29.

Hartmann, Jutta (2012): Improvisation im Rahmen des Zwangs. Gendertheoretische Herausforderungen der Schriften Judith Butlers für pädagogische Theorie und Praxis. In: Balzer, Nicole/Ricken, Norbert (Hrsg.): Judith Butler: Pädagogische Lektüren. Wiesbaden: Springer VS, S. 149-178. https://doi.org/10.1007/978-3-53194368-8_6 
Hartmann, Jutta (2004): Dekonstruktive Perspektiven auf das Referenzsystem von Geschlecht und Sexualität. Herausforderungen der Queer-Theory. In: Glaser, Edith/ Klika, Dorle/Prengel, Annedore (Hrsg.): Handbuch Gender und Erziehungswissenschaft. Bad Heilbrunn: Klinkhardt, S. 255-271.

Hartmann, Jutta (2002): Vielfältige Lebensweisen. Dynamisierungen in der Triade Geschlecht-Sexualität-Lebensform. Kritisch-dekonstruktive Perspektiven für die Pädagogik. Wiesbaden: Springer VS.

Hartmann, Jutta/Holzkamp, Christine/Lähnemann, Lela/Meißner, Klaus/Mücke, Detlef (1998): Lebensformen und Sexualität. Herrschaftskritische Analysen und pädagogische Perspektiven. Bielefeld: Kleine.

Heiner, Maja (2007): Soziale Arbeit als Beruf. Fälle - Felder - Fähigkeiten. München: Ernst Reinhardt.

Heite, Catrin (2009a): Zur Vergeschlechtlichung Sozialer Arbeit im postwohlfahrtsstaatlichen Kontext. Kontinuitäten, Aktualisierungen und Transformationen. In: Kessl, Fabian/Otto, Hans Uwe (Hrsg.): Soziale Arbeit ohne Wohlfahrtsstaat? Zeitdiagnosen, Problematisierungen und Perspektiven. Weinheim: Juventa, S. 100119.

Heite, Catrin (2009b): Soziale Arbeit als Profession im Kontext geschlechterhierarchischer Positionierungen. In: Glaser, Edith/Adresen, Sabine (Hrsg.): Disziplingeschichte der Erziehungswissenschaft als Geschlechtergeschichte. Opladen: Barbara Budrich, S. 49-59.

Helsper, Werner/Tippelt, Rudolf(2011): Ende der Profession oder Professionalisierung ohne Ende? Zwischenbilanz einer unabgeschlossenen Diskussion. In: Dies. (Hrsg.): Pädagogische Professionalität. Weinheim: Beltz, Zeitschrift für Pädagogik Beiheft, 57, S. 268-288.

Helsper, Werner (1996): Pädagogisches Handeln in den Antinomien der Moderne. In: Krüger, Heinz/Helsper, Werner (Hrsg.): Einführung in Grundbegriffe und Grundfragen der Erziehungswissenschaft. Opladen: Barbara Budrich, S. 15-34.

Horstkemper, Marianne/Faulstich-Wieland, Hannelore (1996): Replik. In: Ethik und Sozialwissenschaften. Streitforum für Erwägungskultur 7, 4, S. 578-585.

Huch, Sarah/Lücke, Martin (2015): Sexuelle Vielfalt im Handlungsfeld Schule. Konzepte aus Erziehungswissenschaft und Fachdidaktik. Bielefeld: transcript. https://doi.org/10.14361/9783839429617

Kalthoff, Herbert (2006): Doing undoing class in exklusiven Internatsschulen. In: Georg, Werner (Hrsg.): Soziale Ungleichheit im Bildungssystem. Konstanz: UVK, S. 93-122.

Kampshoff, Marita/Scholand, Barbara (2017): Reflexive Koedukation revisited. In: Dies. (Hrsg.): Schule als Feld - Unterricht als Bühne - Geschlecht als Praxis. Weinheim/Basel: Beltz Juventa, S. 62-81.

Kampshoff, Marita (2013): Doing difference im Unterricht als Unterricht. In: Budde, Jürgen (Hrsg.): Unscharfe Einsätze: (Re-) Produktion von Heterogenität im schulischen Feld. Wiesbaden: Springer Fachmedien, S. 249-274. https://doi.org/10. 1007/978-3-531-19039-6 12

Kerschensteiner, Georg (1921): Die Seele des Erziehers und das Problem der Lehrerbildung, Leipzig: Teubner. https://doi.org/10.1007/978-3-663-16071-7

Kleiner, Bettina/Klenk, Florian C. (2017): Genderkompetenzlosigkeitskompetenz. Grenzen pädagogischer Professionalisierung in der Perspektiver der Queer Theory. 
In: Hartmann, Jutta/Messerschmidt, Astrid/Thon, Christine (Hrsg.): Queertheoretische Perspektiven auf Bildung - Pädagogische Kritik der Heteronormativität. Opladen/Berlin/Toronto: Barbara Budrich, S. 97-119.

Kounin, Jacob S. (1976): Techniken der Klassenführung. Bern/Stuttgart: Huber Klett.

Kuhn, Hans-Peter (2008): Geschlechterverhältnisse in der Schule: Sind die Jungen jetzt benachteiligt? Eine Sichtung empirischer Studien. In: Rendtorff, Barbara/Prengel, Annedore (Hrsg.): Kinder und ihr Geschlecht. Opladen/Farmington Hills: Barbara Budrich, S. 49-71.

Kunert-Zier, Margitta (2005): Erziehung der Geschlechter. Entwicklungen, Konzepte und Genderkompetenz in sozialpädagogischen Feldern. Wiesbaden: Springer VS.

Lang, Juliane/Peters, Ulrich (Hrsg.) (2018): Antifeminismus in Bewegung. Aktuelle Debatten um Geschlecht und sexuelle Vielfalt. Hamburg: MARTA PRESS.

Lipowsky, Frank (2006): Auf den Lehrer kommt es an. Empirische Evidenzen für Zusammenhänge zwischen Lehrerkompetenzen, Lehrerhandeln und dem Lernen der Schüler. In: Allemann-Ghionda, Cristina/Terhart, Ewald (Hrsg.): Kompetenzen und Kompetenzentwicklung von Lehrerinnen und Lehrern. Weinheim/Basel: Beltz, S. 47-70.

Lutz, Helma/ Wenning, Norbert (Hrsg.) (2000): Unterschiedlich verschieden. Differenz in der Erziehungswissenschaft. Opladen

Matzner, Michael/Tischner, Wolfgang (2012): Auf dem Weg zu einer Jungenpädagogik. In: Dies. (Hrsg.): Handbuch Jungen-Pädagogik. 2. Aufl. Weinheim/Basel: Beltz, S. 381-409.

Metz-Göckel, Sigrid/Roloff, Christine (2002): Genderkompetenz als Schlüsselkompetenz. In: Journal Hochschuldidaktik 13，1，S. 7-10. https://doi.org/10.17877/ DE290R-992.

Oevermann, Ulrich (1996): Theoretische Skizze einer revidierten Theorie professionalisierten Handelns. In: Combe, Arno (Hrsg.): Pädagogische Professionalität. Untersuchungen zum Typus pädagogischen Handelns. Frankfurt a.M: Suhrkamp, S. $70-182$.

Offen, Susanne (2017): Eindeutig uneindeutig - Popkulturelle Akteur_innen und Narrationen als Anknüpfungspunkte für die politische Bildung. In: Hartmann, Jutta/Messerschmidt, Astrid/Thon, Christine (Hrsg.): Queertheoretische Perspektiven auf Bildung - Pädagogische Kritik der Heteronormativität. Opladen: Barbara Budrich, S. 121-138.

Ostner, Ilona/Beck-Gernsheim, Elisabeth (1979): Mitmenschlichkeit als Beruf. Eine Analyse in der Krankenpflege. Frankfurt a.M./New York: Campus.

Rendtorff, Barbara/Moser, Vera (1999) (Hrsg.): Geschlecht und Geschlechterverhältnisse in der Erziehungswissenschaft. Eine Einführung. Opladen: Leske + Budrich.

Riegel, Christine (2016): Bildung - Intersektionalität - Othering. Pädagogisches Handeln in widersprüchlichen Verhältnissen. Bielefeld: transcript.

Rieske, Thomas Viola (2011): Bildung von Geschlecht. Zur Diskussion um Jungenbenachteiligung und Feminisierung in deutschen Bildungsinstitutionen. Frankfurt a.M: GEW.

Rousseau, Jean-Jacques (1762/1993): Emile oder Über die Erziehung. Paderborn: Schöningh.

Schneider-Taylor, Barbara (2012): Das ,Band der Frauen` oder Eine Annäherung an Jean-Jacques Rousseaus Frauenbilder. In: Pädagogische Rundschau 66, 3, S. 355365 . 
Schmauch, Ulrike (2016): Sexualpädagogisches Handeln in der Sozialen Arbeit. In: Henningsen, Anja/Timmermanns, Stefan/Tuider, Elisabeth (Hrsg.): Sexualpädagogik kontrovers. Weinheim/München: Beltz Juventa, S. 32-45.

Schmidt Friederike/Schondelmayer, Anne-Christin/Schröder, Ute B. (2015): Selbstbestimmung und Anerkennung sexueller und geschlechtlicher Vielfalt: Lebenswirklichkeiten, Forschungsergebnisse und Bildungsbausteine. Wiesbaden: Springer VS.

Schneider, Claudia (2013): Genderkompetenz: Vom alltagsweltlichen Geschlechterwissen zur theoriegeleiteten Professionalität. In: Ernstson, Sven/Meyer, Christine (Hrsg.): Praxis geschlechtersensibler und interkultureller Bildung. Wiesbaden: Springer VS.

Scholz, Sylka (2010): Hegemoniale Weiblichkeit? Hegemoniale Weiblichkeit! In: Erwägen Wissen Ethik 21, 3, S. 396-398.

Schütz, Berthold (2016): Männlichkeit zwischen Biographie und Profession - Berufliches Selbstverständnis von Jungenarbeitern. Diss. Marburg: Phillips-Universität Marburg.

Spivak, Gayatri C. (1996): The Spivak reader. Landry, Donna/MacLean, Gerald (eds.). New York/London: Routledge.

Spranger, Eduard (1958): Der geborene Erzieher. Heidelberg: Quelle \& Meyer.

Ständige Konferenz der Kultusminister der Länder in der Bundesrepublik Deutschland (KMK) (2004/2014): Standards für die Lehrerbildung: Bildungswissenschaften. Beschluss der Kultusministerkonferenz vom 16.12.2004 i.d.F. vom 12.06.2014. Online unter: http://www.kmk.org/fileadmin/Dateien/veroeffentlichungen_beschluesse/2004/2004_12_16-Standards-Lehrerbildung-Bildungswissenschaften.pdf. [Zugriff: 02.11 1.2018 ].

Terhart, Ewald (2011): Forschung zu Berufsbiographien von Lehrerinnen und Lehrern: Stichworte. In: Terhart, Ewald/Bennewitz, Hedda/Rothland, Martin (Hrsg.): Handbuch der Forschung zum Lehrerberuf. Münster: Waxmann, S. 339-342.

Tervooren, Anja (2006): Im Spielraum von Geschlecht und Begehren. Ethnographie der ausgehenden Kindheit, Weinheim/München: Beltz Juventa..

Timmermanns, Stefan (2003): Keine Angst, die beißen nicht! Evaluation schwul-lesbischer Aufklärungsprojekte an Schulen. Norderstedt: Books on Demand.

Tuider, Elisabeth (2012): Geschlecht und/oder Diversität? Das Paradox der Intersektionalitätsdebatten. In: Kleinau, Elke/Rendtorff, Barbara (Hrsg.): Differenz, Diversität und Heterogenität in erziehungswissenschaftlichen Diskursen. Opladen: Barbara Budrich, S. 79-102.

Walgenbach, Katharina (2014): Heterogenität - Intersektionalität - Diversity in der Erziehungswissenschaft. Opladen: Barbara Budrich.

Westerbarkei, Jan (2014): Intergruppenverhalten. Diskriminierung von Menschen verschiedener sexueller und geschlechtlicher Identitäten. Wiesbaden: Springer VS.

Wigger, Annegret/Weber, Matthias/Sommer, Antje (2012): Ein Pilotprojekt zur Ausbildung Reflexiver Professionalität. In: Becker-Lenz, Roland/Busse, Stefan/Ehlert, Gudrun/Müller-Hermann, Silke (Hrsg.): Professionalität Sozialer Arbeit und Hochschule. Wiesbaden: Springer VS, S. 251-269.

Winker, Gabriele/Degele, Nina (2009): Intersektionalität: Zur Analyse sozialer Ungleichheiten. Bielefeld: transcript. 